\title{
Scoring bias caused by services in table tennis: a statistical analysis
}

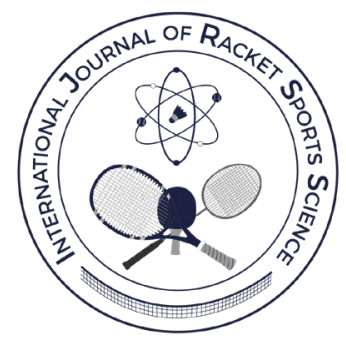

Sho Tamaki', Kazuto Yoshida²

1 Meio University, Japan. s.tamaki@meio-u.ac.jp

2 Juntendo University, Japan. kazuto_yoshida@me.com

Received: 21-05-2020

Accepted: 03-12-2020

\section{Abstract}

In table tennis, servers have the potential advantage of scoring bias when serving. However, the length of the scoring bias, i.e. the shot number where any bias is eliminated, has not been clarified. This study aimed to clarify the level and length of scoring bias occurring at services in table tennis. We analysed 45 men's singles matches (3,665 rallies) and 29 women's singles matches (2,352 rallies) from the 2012 London Olympic games and 49 men's singles matches (4,404 rallies) and 31 women's singles matches (2,320 rallies) from the 2016 Rio Olympic games. The statistical analysis revealed that services generate a low scoring phase at the second shot and slightly high scoring phase at the third shot. Moreover, the level of the scoring bias became trivial after the third shot, although a small scoring bias could remain. Players should therefore be cautious of a scoring bias until the third shot. In the gender comparison, the scoring bias observed in matches of male players was larger than that of female players up to the third shot. This result indicates that male players are more likely to take advantage of service than female players. In the winner/loser comparison, it was found that losers use the service to create scoring bias as effectively as winners do. Losers' inferior skills and tactics in the shots after services were the major factor in the difference in the occurrence of missed shots between winners and losers. Finally, we found that the performance of each shot number should be analysed separately up to the third shot, as the remaining effect of the service is remarkably different among shot numbers. The results of this study contribute important suggestions to the conventional methods of performance analysis that empirically separate a rally into three phases.

Keywords: Table tennis; service; statistical analysis; performance analysis.

Correspondence author: Sho Tamaki, s.tamaki@meio-u.ac.jp

Cite this article as: Tamaki, S. \& Yoshida, K. (2020). Scoring bias caused by services in table tennis: a statistical analysis. International Journal of Racket Sports Science, 2(2), 29-36.

This is an open access article under the CC BY license (https://creativecommons.org/licenses/by/4.0/). 


\section{INTRODUCTION}

In table tennis, the service is different from other shots in terms of the rules and conditions under which it is performed. The laws of table tennis stipulate that "the server shall strike the ball so that it touches first his or her court and then touches directly the receiver's court" (The International Table Tennis Federation, 2019, p.36). In addition, the position of the ball is limited in the laws of table tennis; "from the start of service until it is struck, the ball shall be above the level of the playing surface and behind the server's end line, and it shall not be hidden from the receiver" (The International Table Tennis Federation, 2019, p.36). Another key factor is that the service is performed without any interference from the opponent, whereas the other shots are strongly affected by the opponent's previous shot. As stated above, the rules and condition of service are unique compared to the other shots in a rally.

The unique characteristics of service lead to scoring bias in a rally. Using a statistical analysis of 149 matches in the London Olympics 2012, Yoshida, Yamada, Tamaki, Naito, and Kaga (2014) clarified that the scoring rate, i.e. the percentage of scoring shots to total shots, of servers was higher than that of receivers. The criteria of technique effectiveness proposed by Zhang, Lui, Hu, and Liu (2014) also indicates that servers likely use the advantage of service to score a point. In the criteria, higher effectiveness was required to take high evaluation for the first and the third shot than the second and the fourth shot. Tamaki, Yoshida, and Yamada (2017) analysed scoring bias due to service using the scoring rate and losing rate, i.e. the percentage of missed shots to total shots, of each shot number, which is the number of shot starting from the service, (shot number one is the service, shot number two is the return against the service, and so on). They reported that the number of scoring shots was likely to surpass the number of missed shots at the first shot and the third shot. It can therefore be said that servers have a potential advantage to create a scoring bias for themselves using a service in table tennis.

Though scoring bias affected by services has been investigated by researchers, the length of the scoring bias, i.e. the shot number where any bias is eliminated, has not been clarified. Even if a server has good serving skills and gets high scoring rates at the first and third shots, whether the same conditions remain until the fifth shot or after the fifth shot is unknown because the effect of the service may gradually reduce as the number of shots increases. Tamaki et al. (2017) inferred that the effect of the service does not persist for long in a rally because no significant difference was found in a pairwise comparison of the scoring rate and the losing rate among shot numbers after the third shot. This inference, however, did not clarify the length of the bias because no significant difference does not prove no difference. To the best of our knowledge, there are no studies analysing the length of the scoring bias affected by services other than their work. Thus, the length of scoring bias has not been quantitatively analysed, although researchers assumed or noticed that the bias affected by the service gradually decreases.

If the level and length of the scoring bias can be clarified, we can analyse table tennis more accurately and improve players' tactics. Previous studies implicitly assumed that the length of the effect of service significantly reduces in the early phase of a rally. Wu and Li (1992) evaluated the competitive ability of table tennis players using the occurrence rate or scoring tendency in the three phases in a rally: attack after service (scores at the first or third shot), attack after receive (scores at the second or fourth shot), and rally (scores at the fifth or later shots). This three-phase analysis has been widely adopted in match analyses of table tennis (Fuchs et al., 2018). The method assumes that the scoring bias created by the service decreases significantly after the fourth shot; however, there is no evidence to support this assumption in the papers that adopted this method (Hao, Tian, Hao, \& Song, 2010; Hsu, 2010; Hsu, Chen, \& Wang, 2014; Zhang et al., 2014). If we determined the length of scoring bias affected by the service, the previous method can be revised to analyse table tennis matches more accurately. Knowing the length of scoring bias will also help table tennis players refine their tactics. An effective tactic for a player who is good at service would be to return the player's shot until the level of scoring bias sufficiently decreased. If table tennis players know how many times they have to hit a ball to sufficiently decrease the effect of the service, they can formulate more effective tactics. Thus, the significance of quantifying the length of scoring bias affected by a service is evident from multiple perspectives.

This study therefore aimed to clarify the length of the scoring bias affected by services in table tennis. First, the level of the scoring bias was quantified. The key hypothesis was that the losing rate of a shot would be static after the effect of the service became significantly small. The length of the scoring bias was then estimated according to the change in the level of the scoring bias.

\section{METHOD \\ Match samples}

This study selected 45 men's singles matches (3,665 rallies) and 29 women's singles matches (2,352 rallies) from the matches played at the 2012 London Olympic games and 49 men's singles matches $(4,404$ rallies) and 31 women's singles matches (2,320 rallies) from the 2016 Rio Olympic games. Defensive players were excluded from the sample. Defensive players were identified by their use of long-pimple rubbers, and primary use of chop. Although a four-year time span can change the characteristics of play, we can analyse the scoring biased affected by the service 
commonly observed in 2012-2016 because the service was run under the same rules. The world ranking of the players in the selected matches ranged from 1 to 131, based on the ranking immediately before each Olympic game. The server, winner, and number of the scoring shot were recorded for each rally by observing video recordings broadcasted on television or on the Internet. One unit of the analysis was one player per match. The players were grouped by gender, or match outcome (winners and losers). Written informed consent was unnecessary as the matches were played in public.

\section{Data collection}

The server, winner, and scoring shot number were recorded per rally by observing video recordings broadcast on television or on the Internet. Data were recorded by two operators. If different data were found between the two data collected by the two operators, they reviewed the recordings together, making necessary corrections.

\section{Number of shots, missed shots and scoring shots}

The number of shots, number of missed shots, and number of scoring shots for each server were computed as per the method proposed by Tamaki et al. (2017). In table tennis, players alternate shots. Thus, we can determine which shot a player hit, if we know the server of the rally and the scoring shot number. Let us assume that player A serves to player B, and that the seventh shot scores. We can determine that player A performed the first, third, fifth, and seventh shot; player B thus performed the other shots in the rally, including the eighth shot. In this study, the number of shots is defined as the number of shot opportunities. Therefore, the next from the scoring shot is always counted as the missed shot, regardless of whether it was performed. In the aforementioned example, the eighth shot is the missed shot. By this method, the number of shots, number of missed shots, and number of scoring shots for each server were computed.

\section{Scoring rate and losing rate of each shot number}

We analysed how the services distorted the scoring tendency based on the scoring rate and losing rate. Let ' $\# i$ ' be the $i$-th shot, $n_{i}$ the number of scoring shots at the $i$-th shot, $m_{i}$ the number of missed shots at the $i$-th shot, and $s_{i}$ the number of the $i$-th shot. The scoring rate of the $i$-th shot was calculated by $n_{i} / s_{i}$ and the losing rate of the $i$-th shot was calculated by $m_{i} / s_{i}$. The sixth and subsequent shots by the receiver were unified into a group denoted by ' $\# 6+$ ' and the seventh and subsequent shots from the server were unified into a group denoted by ' $\# 7+$ '. The scoring rate and losing rate were calculated for each player of each match. When $s_{i}$ is small, the scoring rate and losing rate becomes unstable because of a shortage in the sample size. Therefore, if $s_{i}$ was less than 10 , the scoring rate and losing rate of the $i$-th shot were excluded from the statistical analysis. The threshold of $s_{\text {i, }} 10$, was empirically determined based on a balance between statistical stability and the sample size for each shot number. Two samples from \#4, 17 samples from \#5, 19 samples from \#6+, and 55 samples from $\# 7+$ were excluded, because the number of shots were fewer than 10.

\section{Quantification of the level of scoring bias affected by service}

If the scoring bias affected by the service is small, the same losing rate is expected for different shot numbers. The expectation of losing rate $L_{j}^{\prime}$ can be calculated using the following equations:

$$
L_{j}^{\prime}=\sum_{i=j}^{n} m_{i} / \sum_{i=j}^{n} s_{i}
$$

where $n$ is the maximum shot number observed. If the effect of service remains until the $j$-th shot, the observed losing rate becomes farther from $L_{i}^{\prime}$. From another perspective, the distributional distance of the observed losing rate and the expected losing rate can be regarded as the level of scoring bias affected by the service. In this study, the distance between observation and the expected losing rate at the $i$-th shot $D_{i}$ was estimated by applying Cohen's w (Cohen, 1988), a measure of discrepancy between paired proportions. Cohen's $w$ can be calculated using the following equation:

$$
w=\sqrt{\sum \frac{\left(p_{1 i}-p_{0 i}\right)^{2}}{p_{0 i}}}
$$

where $p_{0 i}$ is a proportion posited by the null hypothesis and $p_{1 i}$ is an observed proportion. Sample data are shown in Table 1 and Table 2 to explain how to calculate Cohen's w. Table 1 provides a sample of observed losing rate and returning rate, which is a proportion of the shots other than missed shots calculated by $\left(s_{i}-m_{i}\right) / s_{i}$, for each shot number. Table 2 provides a sample of the losing rate and returning rate expected from specific ranges of shot numbers. The discrepancy between observed and expected proportions at the second shot $D_{2}$ and the third shot $D_{3}$ are approximately 0.4 and 0.22 , which were calculated by the following equations: 


$$
\begin{aligned}
D_{2}= & \frac{(0.08-0.18)^{2}}{0.18}+\frac{(0.14-0.18)^{2}}{0.18}+\frac{(0.2-0.18)^{2}}{0.18}+\frac{(0.23-0.18)^{2}}{0.18}+\frac{(0.26-0.18)^{2}}{0.18} \\
& +\frac{(0.24-0.18)^{2}}{0.18}+\frac{(0.92-0.82)^{2}}{0.82}+\frac{(0.86-0.82)^{2}}{0.82}+\frac{(0.8-0.82)^{2}}{0.82} \\
& \left.+\frac{(0.77-0.82)^{2}}{0.82}+\frac{(0.74-0.82)^{2}}{0.82}+\frac{(0.76-0.82)^{2}}{0.82}\right)^{\frac{1}{2}}
\end{aligned}
$$

$$
\begin{gathered}
D_{3}=\left(\frac{(0.14-0.21)^{2}}{0.21}+\frac{(0.2-0.21)^{2}}{0.21}+\frac{(0.23-0.21)^{2}}{0.21}+\frac{(0.26-0.21)^{2}}{0.21}+\frac{(0.24-0.21)^{2}}{0.21}\right. \\
+\frac{(0.86-0.79)^{2}}{0.79}+\frac{(0.8-0.79)^{2}}{0.79}+\frac{(0.77-0.79)^{2}}{0.79}+\frac{(0.74-0.79)^{2}}{0.79} \\
\left.+\frac{(0.76-0.79)^{2}}{0.79}\right)^{\frac{1}{2}}
\end{gathered}
$$

The expected losing rate depends on the range of shot number used in the calculation; 0.18 is calculated from the second shot and after, and 0.21 is calculated from the third shot and after.

Table 1.

A sample of observed losing rate and returning rate for different shot numbers.

\begin{tabular}{ccccccc}
\hline & \#2 & \#3 & $\mathbf{\# 4}$ & $\mathbf{\# 5}$ & $\mathbf{\# 6 +}$ & $\mathbf{\# 7 +}$ \\
\hline Lossing rate & 0.08 & 0.14 & 0.20 & 0.23 & 0.26 & 0.24 \\
Returning rate & 0.92 & 0.86 & 0.80 & 0.77 & 0.74 & 0.76 \\
\hline
\end{tabular}

Table 2.

A sample of the losing rate and returning rate expected from the specific ranges of shot numbers. For instance, ' $\# 2-\# 7+$ ' denotes that the losing rate and returning rate were calculated from the second shot and after.

\begin{tabular}{lllllll}
\hline $\begin{array}{c}\text { Range of } \\
\text { shot number }\end{array}$ & \#2 & \#3 & \#4 & \#5 & \#6+ & \#7+ \\
\hline$\# 2-\# 7+\quad \begin{array}{l}\text { Losing rate } \\
\text { Returning rate } \\
\text { Losing rate } \\
\text { Returning rate }\end{array}$ & 0.08 & 0.14 & 0.20 & 0.23 & 0.26 & 0.24 \\
R3-\#7+ & 0.86 & 0.80 & 0.77 & 0.74 & 0.76 \\
\hline
\end{tabular}

Cohen (1988) also proposed a general frame of reference for Cohen's $w$ as follows:

Small: $w=0.1$

Medium: $w=0.3$

Large: $w=0.5$

According to Cohen's reference, $D_{2}$ and $D_{3}$ calculated from the sample data shown in Table 1 and Table 2 are regarded as 'between medium and large' and 'between small and medium'. Although Cohen (1998) warned that the magnitude of $w$ should be analysed relatively for a particular problem or field, no better basis for evaluating $w$ than $D_{i}$ was available for use in this study. Therefore, we analysed the magnitude of $D_{i}$ comparatively among shot numbers with reference to the general framework proposed by Cohen.

\section{Statistical analysis}

A Kruskal-Wallis test was performed to compare the scoring rate and losing rate for different shot numbers. Wherever significant differences were observed between the shot numbers, a Dunn test with Bonferroni adjustment was used to compare the shot numbers. The effect size $r$ between shot numbers was calculated by dividing z-test statistic calculated in Dunn test by the square root of the combined sample size (McCarrol, 2016). The scoring rate, losing rate, and scoring bias were compared between male players and female players and between winners and losers with a $95 \%$ confidence interval, calculated by a bootstrap method. Each statistical analysis was tested at a $95 \%$ confidence level.

\section{RESULTS}

\section{Scoring rate and losing rate by shot number}

Fig. 1 shows the distribution of scoring rate and losing rate per shot number and Table 3 shows the effect size between two different shot numbers calculated through the multiple comparisons of scoring rate and losing rate among shot numbers. The scoring rate of shot \#1 was significantly lower than those of the other shot numbers. The scoring rate of shot \#2 was significantly lower than that of \#3, \#4, \#5, and \#6+ in male players' matches; $\# 3, \# 6+$, and $\# 7+$ in female players' matches; $\# 3, \# 4, \# 5, \# 6+$, and $\# 7+$ in winning matches; and \#3 and \#5 in losing matches. The scoring rate of \#3 was significantly higher than $\# 4, \# 6+$, and $\# 7+$ in male players' matches; $\# 4, \# 5$, and $\# 7+$ in female

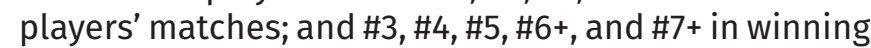
and losing matches. The losing rate of $\# 1, \# 2$, and $\# 3$ were significantly lower than that of the following shot. In the gender comparison, male players' scoring rate was higher than that of female players at shot \#5. In winner and loser comparison, winners' scoring rate was higher than that of losers and winners' losing rate was lower than that of winners at all shot numbers except for \#1 in losing rate.

\section{Distance from the expected distribution of the shot number of missed shots}

Fig. 2 shows the distributional distance between observed losing rate and expected losing rate. As the shot number increased, the distance gradually grew smaller. In the gender comparison, $D_{2}$ of female players was lower than that of male players. No significant differences were found in comparison between winners and losers. 


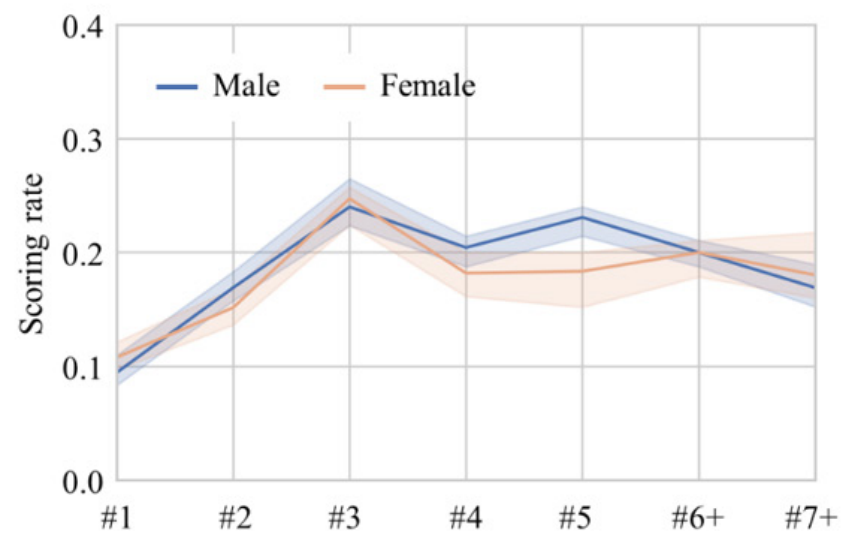

(a)

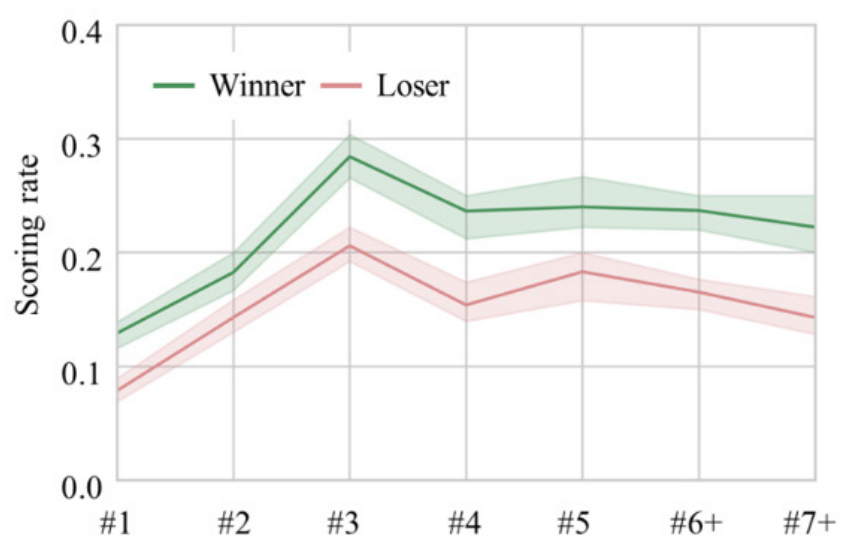

(c)

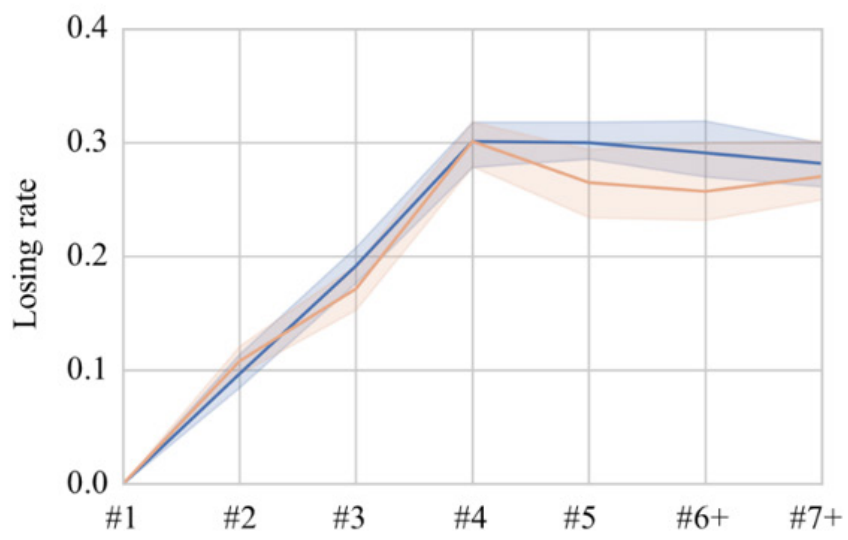

(b)

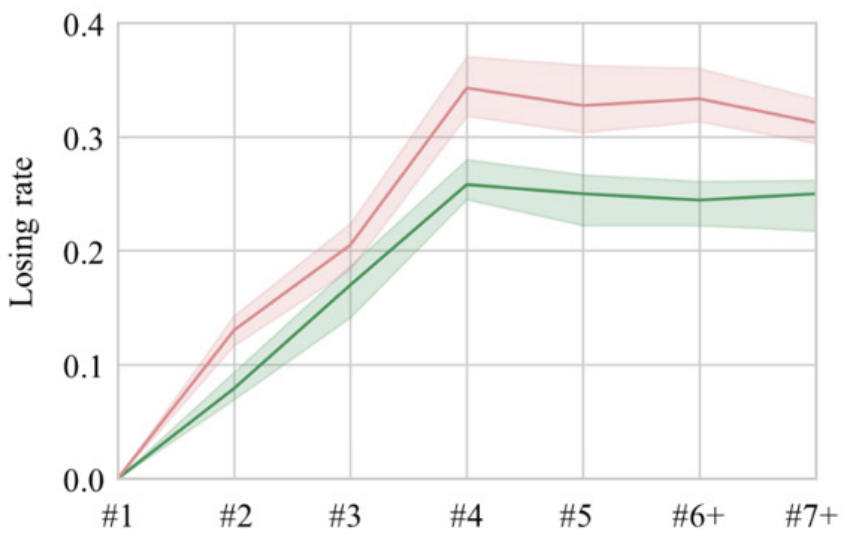

(d)

Figure 1. Scoring rate and losing rate at each shot number. The median of scoring rate and losing rate was calculated for each gender, winners, and losers. The shaded area denotes the $95 \%$ confidence interval.

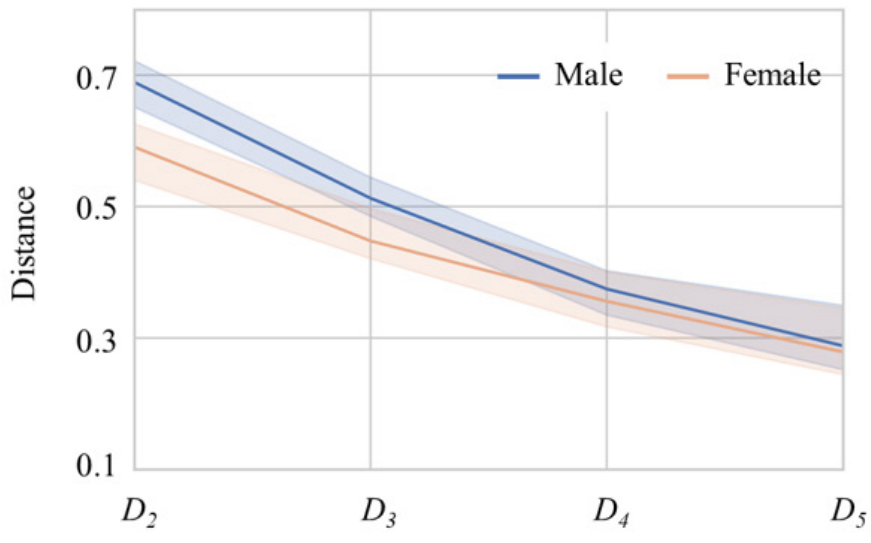

(a)

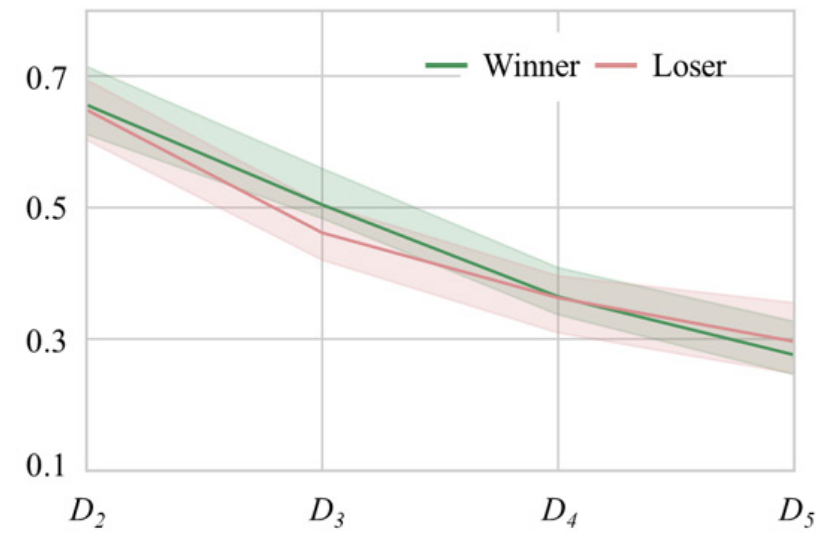

(b)

Figure 2. Distances between the observed losing rates and the expected losing rates at each shot number. $\mathrm{D}_{\mathrm{i}}$, the distributional distance at $\mathrm{i}$-th shot, was calculated from the $\mathrm{i}$-th and subsequent shots. The median of the scoring rate and the losing rate was calculated for each gender and for winners and losers. The shaded area denotes the $95 \%$ confidence interval. 
Table 3.

Effect size between two different shot numbers calculated through the multiple comparisons of scoring rate and losing rate based on shot numbers. The tables show the results for each gender and for winners and losers. The filled colour of each cell denotes the corrected p-value calculated by pairwise comparison as described at the bottom of the table with pink cells denoting that the shot indicated at the left of the table is greater than the shot indicated at the top of the table (denoted as left > top) while the blue cells indicate that the shot at the left of the of the table is less than that at the top of the table (left < top). The effect size in uncoloured cells is not sianificant.

\begin{tabular}{|c|c|c|c|c|c|c|c|c|c|c|c|c|c|c|c|c|}
\hline & \multicolumn{8}{|c|}{ Scoring rate } & \multicolumn{8}{|c|}{ Losing rate } \\
\hline & & $\# 1$ & $\# 2$ & $\# 3$ & $\# 4$ & $\# 5$ & $\# 6+$ & $\# 7+$ & & $\# 1$ & $\# 2$ & $\# 3$ & $\# 4$ & $\# 5$ & $\# 6+$ & $\# 7+$ \\
\hline \multirow{7}{*}{$\frac{2}{2}$} & $\# 1$ & & 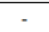 & 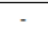 & - & - & 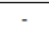 & 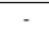 & $\# 1$ & & - & - & - & - & - & - \\
\hline & $\# 2$ & 0.34 & & - & - & - & - & - & $\# 2$ & 0.35 & & - & . & - & - & - \\
\hline & $\# 3$ & 0.72 & 0.38 & & - & - & - & - & $\# 3$ & 0.65 & 0.31 & & - & - & - & - \\
\hline & $\# 4$ & 0.53 & 0.19 & 0.19 & & - & - & - & $\# 4$ & 1.06 & 0.71 & 0.41 & & - & - & - \\
\hline & $\# 5$ & 0.63 & 0.29 & 0.09 & 0.10 & & - & - & $\# 5$ & 1.05 & 0.71 & 0.40 & 0.01 & & - & - \\
\hline & $\# 6+$ & 0.50 & 0.17 & 0.22 & 0.03 & 0.13 & & - & $\# 6+$ & 1.04 & 0.69 & 0.39 & 0.02 & 0.01 & & - \\
\hline & $\# 7+$ & 0.38 & 0.04 & 0.33 & 0.15 & 0.25 & 0.12 & & $\# 7+$ & 0.98 & 0.63 & 0.33 & 0.08 & 0.07 & 0.06 & \\
\hline
\end{tabular}

\begin{tabular}{|c|c|c|c|c|c|c|c|c|c|c|c|c|c|c|}
\hline$\# 1$ & $\# 2$ & $\# 3$ & $\# 4$ & $\# 5$ & $\# 6+$ & $\# 7+$ & & $\# 1$ & $\# 2$ & $\# 3$ & $\# 4$ & $\# 5$ & $\# 6+$ & $\# 7+$ \\
\hline & - & - & - & - & - & - & $\# 1$ & & - & - & - & - & - & - \\
\hline 0.21 & & - & - & - & - & - & $\# 2$ & 0.41 & & - & - & - & - & - \\
\hline 0.69 & 0.48 & & - & - & - & - & $\# 3$ & 0.64 & 0.22 & & - & - & - & - \\
\hline 0.37 & 0.16 & 0.32 & & - & - & - & $\# 4$ & 1.10 & 0.69 & 0.47 & & - & - & - \\
\hline 0.36 & 0.15 & 0.33 & 0.01 & & - & - & $\# 5$ & 0.97 & 0.55 & 0.33 & 0.13 & & - & - \\
\hline 0.49 & 0.28 & 0.20 & 0.12 & 0.13 & & - & $\# 6+$ & 1.00 & 0.59 & 0.36 & 0.10 & 0.03 & & - \\
\hline 0.42 & 0.21 & 0.27 & 0.05 & 0.06 & 0.07 & & $\# 7+$ & 1.02 & 0.61 & 0.39 & 0.08 & 0.06 & 0.02 & \\
\hline
\end{tabular}

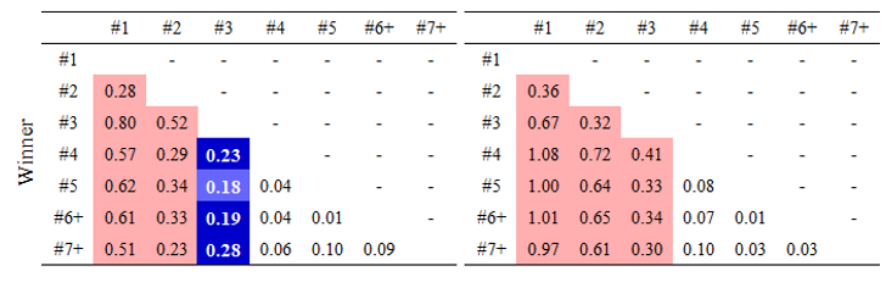

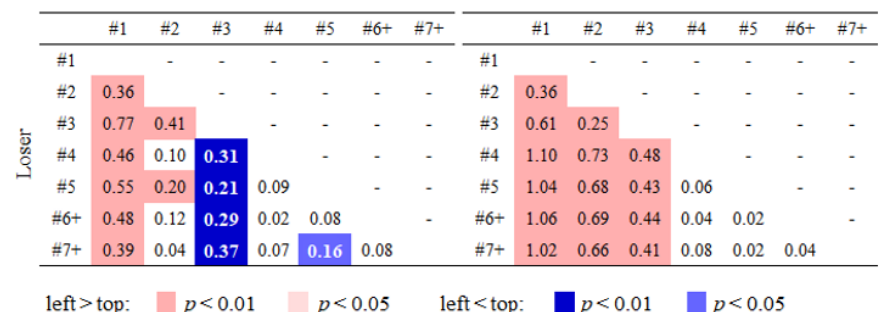

\section{DISCUSSION}

\section{Scoring bias affected by service}

Service was found to lower the scoring rate and losing rate of shot $\# 2$, lower the losing rate and increase the scoring rate compared to other shots. It is possible that the low losing rate on shot $\# 2$ could be due to the difficulty in scoring from \#1, which is, in turn, due to the laws of table tennis as mentioned in the introduction. The low scoring rate of $\# 2$ cannot be described by the low scoring rate or low losing rate of $\# 1$. The low scoring rate of $\# 1$ and high scoring rate of \#2 could be co-occurring; moreover, the tactics of servers may affect the scoring rate of $\# 2$. Considering the difficulty in scoring at service, servers may make it difficult for receivers to score on their return, which may be achieved by skilfully leading opponents to incorrect identification of the spin or placement of a service (Geske \& Mueller, 2010, pp.71-77). As the receiver is required to penetrate such deception by the server, the scoring rate of $\# 2$ was commonly lower than that of the following shots. The low losing rate of $\# 3$ could be the result of the low scoring rate of $\# 2$. The high scoring rate of $\# 3$, however, does not result from the low scoring rate or low losing rate of $\# 2$. As with the low scoring rate of $\# 2$, the high scoring rate of $\# 3$ could be due to the servers' skills, such as intentionally misleading opponents in their identification of the spin and the placement of service. In terms of effect size, however, the difference was relatively small, particularly in female players and winners. We can therefore conclude that the service was mainly found to generate a low scoring phase at shot $\# 2$ and relatively small effect at \#3 as the server's scoring bias.

\section{How does the level of scoring bias change with each shot?}

It was found that the level of scoring bias became insignificant from shot \#4, although a slight scoring bias could remain. According to Fig. 2, scoring bias decreased drastically with each shot. Moreover, Table 3 shows that the effect size was remarkably small in the comparison of scoring rate and losing rate among all shots after \#3. These results indicate that the scoring rate became smaller every time a shot was performed. According to Cohen's reference, however, $D_{4}$ and $D_{5}$ can be interpreted as medium. Although we should avoid placing too much importance on Cohen's criteria, the observed distance indicates that a certain level of scoring bias could remain, even at shot \#4 and at subsequent shots. However, the scoring bias would be too trivial to consider if its effect on scoring rate and losing rate became small. According to the effect size of scoring rate and losing rate between shot numbers (Table 3), we can estimate that the level of the remaining scoring bias would be small for the shots after \#3. We should therefore be cautious of scoring bias after \#3 only if we know that the latter phase of a rally is important for the analysis of matches.

Quantifying the level of scoring bias affected by service was a notable achievement of the current study. In the results of their pair-wise comparison of losing rate, Tamaki et al. (2017) implied that the effect of service became small after \#3 because there were no significant differences among shot numbers after shot \#3. However, the absence of significant difference does not imply no difference at all. Moreover, they could not determine whether a small scoring bias would remain after \#3 because the level of scoring bias was not quantified. In the current study, the level of scoring bias was calculated based on the distributional distance from the expected losing rate, which allowed us to understand the changes in scoring bias at each shot.

\section{Gender comparison}

The level of scoring bias in the early phase of a rally differed between genders. In the male players' matches, the impact of service was greater than that in female 
players' matches. From this result, we can hypothesise that male players are more likely to take advantage of service than female players. However, the difference between genders reduced at every shot and became little or none from shot \#4. These results suggest that the level of scoring bias is different between genders particularly up to \#3. These results also suggest that the level of scoring bias is not strongly related to its sustainability. Even if some servers have good serving skills, the length of scoring bias would be virtually the same as the common length.

\section{Winner and loser comparison}

Identical scoring bias was observed in winning and losing matches, whereas the scoring rate and losing rate were evidently different. When comparing the scoring rate and the losing rate between the winners and losers, the winners seem to use the service more effectively than the losers. However, no difference in scoring bias was found between the winners and losers. Let us compare the main effect of service specifically, lowering the scoring rate at \#2- between winners and losers. The average effect size between $\# 2$ and at each shot after \#2 of winners was $0.34( \pm 0.11)$ and that of losers was $0.17( \pm 0.14)$. As winners' shot \#2 was affected by losers' services, the results may indicate that losers service lowered the scoring rate at shot \#2 more than winners' service did. However, it should be noted that winners' scoring rate of shots after the \#2 was significantly higher than that of losers. It is also possible that winners' superior skills or tactics at the shots after \#3 increased the difference between scoring rate at \#2 and that of other shots. Although this cannot simply be concluded that from the results, it can be said that losers' service evidently biased the scoring tendency at least as much as the winners' service did. Next, we discuss the difference in the shots after the service. Because the scoring bias has been reduced in the shots after \#3, the difference in the losing rate after \#3 can be a measure of differences in tactics or hitting skills at the shots after the service. The confidence interval of losing rate after \#3 was $[0.24,0.26]$ in winning matches, and [0.33, 0.35 ] in losing matches. A rough calculation shows the difference between the two intervals to be 0.09. The losers' inferior skills and tactics at the shots after the service increases the differences on the losing rate at all shots after the service between winners and losers. Therefore, losers use the service effectively and create a certain level of scoring bias in a rally at least as well as winners do; however, the scoring rate is low and losing rate is high because of the poor performance of the shots after the service.

\section{Suggestions for the methods of performance analysis}

Based on our results, we suggest that the performance of table tennis players should be analysed according to each separate shot number, particularly up to \#4. The method proposed by Wu and Li (1992), which has been widely adopted in table tennis, separates a rally into the following three phases: shots $\# 1$ and \#3, shots \#2 and \#4, and shots after \#4. This analysis method is reasonable for analysing players' total performance of serving skill or returning skill; however, the performance analysis would be more accurate if we separate \#1 and \#3 and \#2 and \#4. This is because the remaining level of scoring bias is remarkably different among shot numbers up to \#4. Furthermore, unifying the shots after \#4 was validated to be reasonable in most cases in real life scenarios. However, we should consider separating the shots after \#4 if we know important features that exist in the latter phase of a rally.

\section{Limitations of this study}

The findings of this study should be considered along with its limitations. As this study focused on the average effect of the service in table tennis, the scoring bias affected by the shots after the service were analysed separately. In the rally of table tennis, however, every shot may change the scoring tendency and create the scoring bias. It should be noted that the findings of this study are approximations calculated under the ideal hypothesis that the losing rate of a shot is static after the effect of the service became significantly small. Moreover, the scoring bias affected by service may change over time. In this study, the period of time we selected the matches from was 2012 to 2016. As the techniques and tactics of table tennis are constantly changing, the bias in scoring by service will not necessarily be the same in the future. It is necessary to study the scoring bias affected by service at regular intervals in order to accurately analyse table tennis matches and construct effective tactics. In addition, it should be noted that it is very difficult to analyse the variation in the effect of services at the shots after \#5 through this research methodology. Since offensive styles have become dominant in modern table tennis, scores are often decided early in a rally. Therefore, it is hard to collect enough information on the long-lasting phase of a rally. Although we clarified that a slight scoring bias could remain even after shots \#4, the variation of the effect of services after the shots after \#4 could not be mentioned.

\section{CONCLUSION}

This study investigated the level and length of scoring bias affected by service in table tennis. We found that services generate a low scoring phase at the second shot and slightly high scoring phase at the third shot. Moreover, the level of scoring bias was revealed to become trivial at all shots after the third shot, although a slight scoring bias could remain. The scoring rate was estimated from the distributional distance between the observed losing rate of each shot number and the estimated losing rate. The differences in scoring bias between male players and 
female players and between winners and losers were also observed. In the gender comparison, the scoring bias observed in male players' matches was larger than that observed in female players' matches up to the third shot. In the winner and loser comparison, it was found that losers also use service effectively and create a certain level of scoring bias as much as winners do. Finally, we suggested that the performance of each shot number should be analysed separately up to the third shot.

\section{DISCLOSURE STATEMENT}

The authors report no conflicts of interest

\section{REFERENCES}

Cohen, J. (1988). Statistical power analysis for the behavioral sciences (2nd ed.). New Jersey: Lawrence Erlbaum Associates.

Fuchs, M., Liu, R., Malagoli Lanzoni, I., Munivrana, G., Straub, G., Tamaki, S., Yoshida, K., Zhang, H., \& Lames, M. (2018). Table tennis match analysis: a review. Journal of Sports Sciences, 36, 2653-2662. http://dx.doi.org/10.1080/02640414.2018.1450073

Geske, K. M., \& Mueller, J. (2010). Table tennis tactics your path to success. Aachen: Meyer \& Meyer Sport.

Hao, Z., Tian, Z., Hao, Y., \& Song, J. (2010). Analysis on technique and tactics of Lin $\mathrm{Ma}$ and Hao Wang in the men's single table tennis final in the 29th Olympic Games. International Journal of Table Tennis Sciences, 6, 74-78.

Hsu, M.-H. (2010). A study on the technical analysis and attack-defense performance of men's top four single players in 2008 Olympic Games. International Journal of Table Tennis Sciences, 6, 248-260.
Hsu, M.-H., Chen, Y.-F., \& Wang, S.-C. (2014). Offense defense mode analysis of the world top male table tennis player - A case study by Chuang Chi-Yuan who participated in 2012 London Olympic Male Single Games. Journal of Science and Innovation, 4(2), 41-50.

McCarrol, D. (2016). Simple Statistical Tests for Geography. New York: CRC Press. http://dx.doi. org/10.1201/9781315380438

Tamaki, S., Yoshida, K., \& Yamada, K. (2017). A shot number based approach to performance analysis in table tennis. Journal of Human Kinetics, 55, 7-18. http://dx.doi.org/10.1515/hukin-2017-0002

The International Table Tennis Federation (2019). Handbook 2019 (pp.36-37). Lausanne: The International Table Tennis Federation.

Wu, H., \& Li, Z. (1992). Research on technical diagnosis method for table tennis players. International Journal of Table Tennis Sciences, 1, 99-103.

Yoshida, K., Yamada, K., Tamaki, S., Naito, H., \& Kaga, M. (2014). Characteristics of world-class rally in table tennis: focusing on the number of shots played per rally. The Japan Journal of Coaching Studies, 28(1), 65-74.

Zhang, H., Liu, W., Hu, J.-J., \& Liu, R.Z. (2014). Evaluation of elite table tennis players' technique effectiveness. Journal of Sports Sciences, 32(1), 70-77.

http://dx.doi.org/10.1080/02640414.2013.792948 